\title{
PERCEPCIÓN DE LOS PROFESIONALES GINECO OBSTETRAS Y \\ OBSTETRAS DE LA ATENCIÓN CON ENFOQUE INTERCULTURAL A LAS PACIENTES OBSTÉTRICAS EN EL AMBITO DE LA CIUDAD DE TACNA
}

\author{
Responsable : Lic.obst. Leandra Llanca Ramos \\ Miembros : Lic.obst. Patricia Ramos Huajardo \\ Lic. Enf. Dalma Torres Guzman
}

\section{RESUMEN}

La presente investigación realizada permitió determinar la percepción de los profesionales gineco-obstetras y obstetras de la atención con enfoque intercultural a las pacientes obstétricas, en el ámbito de la ciudad de Tacna. Para tal efecto se entrevistaron y encuestaron a 18 médicos Ginec-obstetras y 100 obstetras que laboran en los diferentes establecimientos de salud de nuestra ciudad. Los resultados obtenidos nos indican que los profesionales de salud reconocen carecer en su formación universitaria de elementos interculturales y, a pesar de elic, consideran que la atención con este enfoque es un derecho, una necesidad y que se justifica su implementación. Por otra parte, reconocen que la condición étnica genera inequidad $y$ discriminación en la atención de salud.

\section{ABSTRACT}

The following investigation allowed to determine the perception of the Gineco-obstetras and obstetricians in the attention with intercultural approach to the obstetrical patients, in the scope of the city of Tacna. For such effect 18 GynecoObstetrician and 100 Obstetricians that work in different Health Establishments in our city were interviewed. The obtained results indicate that health professionals recognize the lack of intercultural elements in their professional formation and they consider that the attention with this approach is a right, a necessity and that its implementation is justified. On the other hand they recognize that the ethnic condition generates inequality and discrimination in the health attention.

\section{INTRODUCCIÓN}

En las últimas décadas, la interculturalidad en salud ha sido un concepto para designar al conjunto de acciones y políticas que tienden a conocer e incorporar la cultura del usuario en el proceso de atención de salud. Pues el tema de la pertinencia cultural del proceso de atención es un fenómeno que trasciende lo exclusivamente étnico pues implica valorar la diversidad biológica, cultural y social del ser humano como un factor importante en todo proceso de salud y enfermedad.

El respeto a esta diversidad tiene larga trayectoria en paises cuyas altas tasas de inmigración han generado contextos de gran diversidad étnica y cultural, situación que ha puesto al equipo de salud en el imperativo de desarrollar estrategias que faciliten la relación médico-paciente. La necesidad de desarrollar procesos interculturales en salud se ha generado en los paises latinoamericanos por diversas razones históricas, sociopolíticas y epidemiológicas, las cuales han motivado iniciativas tendientes a evitar que la identidad étnica y cultural del usuario constituya una barrera en el acceso y oportunidad a una mejor atención de salud. Nuestro país cuenta con diversos grupos étnicos, lo que le da un carácter pluricultural y nace la imperiosa necesidad de ponernos a trabajar con un enfoque o modelo que nos permita contemplar las diversas culturas poblacionales en la atención de salud.

Las diferencias culturales entre pacientes y profesionales se observan también al interior de los grupos, lo cual refleja el carácter dinámico de la cultura. Como toda construcción humana, los componentes cognitivos y materiales se transforman en la medida que el grupo social experimenta nuevos desafíos y problemas. La biomedicina y las culturas médicas tradicionales experimentan transformaciones en sus modelos explicativos de la enfermedad e incorporan nuevas tecnologias y replantean roles profesionales, técnicos 0 empiricos para abordar aspectos de salud.

El concepto de cultura aplicado al campo de la salud se constituye en un eje neurálgico en el análisis de los factores sociales involucrados en cualquier proceso de salud y enfermedad. El reconocimiento de las diferencias y similitudes culturales entre usuarios y proveedores nos permite comprender el nivel y carácter de las expectativas que cada cual tiene del proceso, las percepciones de salud y enfermedad representadas en la interacción médicopaciente, los patrones con que se evalúa la calidad y resultado de la acción terapéutica, y el despliegue de diversas conductas frente a la enfermedad.

En la atención materna se han encontrado diferentes obstáculos, son las denominadas Barreras Culturales. No hemos entendido que la forma de pensar, sentir y vivir es diferente. Ha sido dificil desarrollar y sostener estrategias de adecuación cultural, sobre todo en el campo de la atención materna, lo que ha determina que un gran grupo no acceda a los servicios de salud, sencillamente porque ellos no se adecuan a sus patrones culturales y debemos tener presente que la salud es parte de la cultura de un pueblo, de un país.

\section{OBJETIVO}

Determinar la percepción de la Interculturalidad en salud de los profesionales gineco-obstetras y obstetras de los Centros Hospitalarios y Centro de Atención Primaria de la ciudad de Tacna en el año 2006. 


\section{MATERIALY MÉTODO}

\section{1) Lugar de Ejecución}

El presente trabajo se realizó con la finalidad de determinar la percepción de la Interculturalidad en salud de los profesionales gineco - obstetras y obstetras de los Centros Hospitalarios y Centros de Atención Primaria en el ámbito de la ciudad de Tacna.

\section{2) Muestra de Estudio}

Constituida por médicos gineco-obstetras y obstetras de la ciudad de Tacna que corresponden a 30 médicos y 250 obstetrices que laboran actualmente bajo diferentes modalidades en los Centros Hospitalarios y de atención primaria de la ciudad de Tacna en el año 2006. De lo cual se tomó como muestra el $40 \%$ de dicha población

\section{3) Recolección de Datos}

Para el registro de información se utilizó como instrumento una matriz de recolección de datos recabados a través de la encuesta-entrevista.

\section{4) Procesamiento y Análisis de Datos}

Se utilizó: Procesador de Textos Microsoft Word,Programa Excell,Programa Epi-Info

\section{RESULTADOS}

Según el Cuadro N'01, la mayor parte de profesionales no han recibido formación con elementos interculturales constituyendo un $32,5 \%$.

Cuadro N 01: Por Estudio de Acuerdo con Formación Profesional en Relación con Elementos de Interculturalidad

\begin{tabular}{|l|r|c|r|r|r|c|}
\hline \multirow{2}{*}{ Elementos } & \multicolumn{2}{|c|}{ Si } & \multicolumn{2}{c|}{ No } & \multicolumn{2}{c|}{ Total } \\
\cline { 2 - 7 } & \multicolumn{1}{|c|}{$\mathbf{N}^{\circ}$} & \multicolumn{1}{c|}{$\%$} & $\mathbf{N}^{\circ}$ & $\%$ & $\mathbf{N}^{\circ}$ & \multicolumn{1}{c|}{$\%$} \\
\hline Idioma extranjero (Inglès) & 12 & $3,4 \%$ & 106 & $29,9 \%$ & 118 & $33,3 \%$ \\
\hline Dialecto & 3 & $0,8 \%$ & 115 & $32,5 \%$ & 118 & $33,3 \%$ \\
\hline Medicina tradicional & 32 & $9,0 \%$ & 86 & $24,3 \%$ & 118 & $33,3 \%$ \\
\hline Total & 47 & $13,3 \%$ & 307 & $86,7 \%$ & 354 & $100 \%$ \\
\hline
\end{tabular}

Fuente: Encuesta-entrevista realizada a los profesionales de salud: ginecólogos y obstetras u obstetrices de la localidad de Tacna.

Los profesionales consideran que es un derecho, una necesidad. Se justifica la atención con enfoque intercultural, en un $24,2 \%, 25,0 \%, 24,4$ y $15,3 \%$, respectivamente. Sólo el $15,3 \%$ señala que la población debe adaptarse a los servicios de salud.

Cuadro $\mathbf{N}^{\circ}$ 02:Percepción de la Atención con Enfoque Interculturalidad

\begin{tabular}{|l|c|c|r|r|r|r|}
\hline \multirow{2}{*}{ Percepción } & \multicolumn{2}{|c|}{ Si } & \multicolumn{2}{c|}{ No } & \multicolumn{2}{c|}{ Total } \\
\cline { 2 - 7 } & $\mathbf{N}^{\circ}$ & \multicolumn{1}{c|}{$\%$} & $\mathbf{N}^{\circ}$ & $\%$ & $\mathbf{N}^{\circ}$ & $\%$ \\
\hline Derecho de la población & 114 & $24,2 \%$ & 4 & $0,8 \%$ & 118 & $25,0 \%$ \\
\hline $\begin{array}{l}\text { Necesidad para facilitar el } \\
\text { acceso a servicio de salud }\end{array}$ & 118 & $25,0 \%$ & 0 & $0,0 \%$ & 118 & $25,0 \%$ \\
\hline $\begin{array}{l}\text { Se justifica su } \\
\text { implementación }\end{array}$ & 115 & $24,4 \%$ & 3 & $0,6 \%$ & 118 & $25,0 \%$ \\
\hline $\begin{array}{l}\text { La población debe adap- } \\
\text { tarse a los servicios de } \\
\text { salud que se les brinda }\end{array}$ & 72 & $15,3 \%$ & 46 & $9,7 \%$ & 118 & $25,0 \%$ \\
\hline \multicolumn{1}{|c|}{ Total } & 419 & $88,8 \%$ & 53 & $11,2 \%$ & 472 & $100 \%$ \\
\hline
\end{tabular}

Fuente: Encuesta-entrevista realizada a los profesionales de salud: ginecólogos y obstetras u obstetrices de la localidad de Tacna.

La mayoria de profesionales considera que existe inquietud, discriminación y falta de conccimiento de las culturas quechuas y aymaras en un $27,1 \%, 21,5 \%$ y $27,7 \%$, respectivamente.

Cuadro Nº3: Percepción de los Profesionales de la Salud de la Influencia de la Condición Étnica en la Calidad de Atención

\begin{tabular}{|l|c|c|c|c|c|c|}
\hline \multicolumn{1}{|c|}{$\begin{array}{c}\text { Situaciones que } \\
\text { genera la } \\
\text { condición étnica }\end{array}$} & \multicolumn{2}{|c|}{ Si } & \multicolumn{2}{|c|}{ No } & \multicolumn{2}{c|}{ Total } \\
\cline { 2 - 7 } & $\mathbf{N}^{0}$ & $\%$ & $\mathbf{N}^{0}$ & $\%$ & $\mathbf{N}^{\circ}$ & $\%$ \\
\hline $\begin{array}{l}\text { La condición étnica } \\
\text { genera inseguridad }\end{array}$ & 96 & $27,1 \%$ & 22 & $6,2 \%$ & 118 & $33,3 \%$ \\
\hline $\begin{array}{l}\text { Hay discriminación del } \\
\text { equipo de salud a la } \\
\text { población indigena }\end{array}$ & 76 & $21,5 \%$ & 42 & $11,9 \%$ & 118 & $33,3 \%$ \\
\hline $\begin{array}{l}\text { Falta de conocimiento } \\
\text { sobre la cultura aymará } \\
\text { y quechua }\end{array}$ & 98 & $27,7 \%$ & 20 & $5,6 \%$ & 118 & $33,3 \%$ \\
\hline \multicolumn{1}{|c|}{ Total } & 270 & $76,3 \%$ & 84 & $23,7 \%$ & 354 & $100 \%$ \\
\hline
\end{tabular}

Fuente: Encuesta-entrevista realizada a los profesionales de salud: ginecólogos y obstetras u obstetrices de la localidad de Tacna.

El elemento que mayormente dificulta el acceso de la población indigena a los servicios de salud es la falta de preparación para brindar atención con enfoque intercultura constituyendo un $25,1 \%$, seguido del maltrato en la atención con un $24,6 \%$ y en un tercer lugar los mitos y creencias de la población con un $19.9 \%$

Cuadro $N^{\circ}$ 04: Percepción de los Profesionales sobre los Elementos que Dificultan el Acceso de la Población Indigena a los Servicios de Salud

\begin{tabular}{|l|c|c|}
\hline \multicolumn{1}{|c|}{ Elementos } & $\mathbf{N}^{0}$ & $\%$ \\
\hline Idioma & 30 & $7,7 \%$ \\
\hline Mitos y creencias de la población & 78 & $19,9 \%$ \\
\hline Maltrato por parte del personal de salud & 96 & $24,6 \%$ \\
\hline Idiosincrasia de la población & 47 & $12,0 \%$ \\
\hline $\begin{array}{l}\text { Falta de preparación profesional para brindar } \\
\text { atención con enfoque intercultural }\end{array}$ & 98 & $25,1 \%$ \\
\hline Incomprensión & 42 & $10,7 \%$ \\
\hline \multicolumn{1}{|c|}{ Total } & 391 & $100 \%$ \\
\hline
\end{tabular}

Fuente: Encuesta-entrevista realizada a los profesionales de salud: ginecólogos y obstetras u obstetricias de la localidad de Tacna.Se puede apreciar en el siuiente cuadro que en un $92,4 \%$ conoce las normas técnicas del MINSA y sólo el 7,6\% la desconoce.

Cuadro N 05: Por el Estudio de Acuerdo al Grado de Conocimiento de las Normas Técnicas del Minsa para la Atención del Parto Vertical

\begin{tabular}{|l|r|r|}
\hline \multicolumn{1}{|c|}{ Grado de conocimiento } & $\mathbf{N}^{\circ}$ & \multicolumn{1}{c|}{$\%$} \\
\hline Conoce totalmente & 78 & $66,1 \%$ \\
\hline Conoce parcialmente & 31 & $26,3 \%$ \\
\hline Desconoce & 9 & $7,6 \%$ \\
\hline \multicolumn{1}{|c|}{ Total } & 118 & $100 \%$ \\
\hline
\end{tabular}


Fuente: Encuesta - entrevista realizada a los profesionales de salud: ginecólogos y obstetras u obstetrices de la localidad de Tacna.

Se puede apreciar en el cuadro que en un $97,5 \%$ no se ha implementado la atención del parto vertical, sólo en un $2,5 \%$ se ha implementado parcialmente.

Cuadro $\mathbf{N}^{\circ}$ 06: Población en Estudio de Acuerdo a Implementación de la Atención del Parto Vertical en sus Instituciones de Origen

\begin{tabular}{|c|c|c|}
\hline Implementación & $\mathbf{N}^{\circ}$ & $\%$ \\
\hline $\mathrm{Si}$ & 0 & $0,0 \%$ \\
\hline Parcial & 3 & $2,5 \%$ \\
\hline No & 115 & $97,5 \%$ \\
\hline Total & 118 & $100 \%$ \\
\hline
\end{tabular}

Fuente: Encuesta-entrevista realizada a los profesionales de salud: ginecólogos y obstetras u obstetrices de la localidad de Tacna. El factor que más influye es la falta de implementación para la atención del parto vertical con enfoque intercultural es la falta de capacitación con $28,3 \%$, seguido de la inadecuada infraestructura con un $26,6 \%$, también tiene un porcentaje significativo la falta de implementación de las guias de manejo con un $25,2 \%$.

Cuadro N 07: Población en Estudio de Acuerdo con Causas de la Falta de Implementación del Parto Vertical y Enfoque Intercultural en la Atención Obstétrica

\begin{tabular}{|l|r|c|}
\hline \multicolumn{1}{|c|}{ Causas } & $\mathbf{N}^{\circ}$ & $\%$ \\
\hline Inadecuada infraestructura & 96 & $26,6 \%$ \\
\hline Falta de capacitación & 102 & $28,3 \%$ \\
\hline Falta de motivación & 72 & $19,9 \%$ \\
\hline $\begin{array}{l}\text { Falta de implementación de las guias de manejo } \\
\text { para la atención del parto vertical }\end{array}$ & 91 & $25,2 \%$ \\
\hline \multicolumn{1}{|c|}{ Total } & 361 & $100 \%$ \\
\hline
\end{tabular}

Fuente: Encuesta-entrevista realizada a los profesionales de salud: ginecólogos y obstetras u obstetrices de la localidad de Tacna.

El mayor porcentaje de la población está de acuerdo con el acompañamiento familiar en el parto con un $77,1 \%$ que es muy significativo.

Cuadro N 08: Población en Estudio de Acuerdo si el Profesional de Salud Permitiria el Acompañamiento Familiar Durante el Parto.

\begin{tabular}{|c|c|c|}
\hline $\begin{array}{c}\text { Permitiria el acompañamiento } \\
\text { familiar durante el parto }\end{array}$ & $\mathbf{N}^{\circ}$ & $\%$ \\
\hline Si & 91 & $77.1 \%$ \\
\hline No & 27 & $22.9 \%$ \\
\hline Total & 118 & $100 \%$ \\
\hline
\end{tabular}

Fuente: Encuesta-entrevista realizada a los profesionales de salud: ginecólogos y obstetras u obstetrices de la localidad de Tacna.

En un $64.4 \%$ los profesionales están de acuerdo en optar por otras posiciones para la atención del parto y sólo el $35.6 \%$ no está de acuerdo.

Cuadro No 09: Población en Estudio de Acuerdo si el
Profesional de Salud Aceptaria que la Madre Opte por Otras Posiciones en laAtención del Parto.

\begin{tabular}{|c|c|c|}
\hline Acoptación & $\boldsymbol{N}^{\circ}$ & $\%$ \\
\hline $\mathrm{Si}$ & 76 & $64.4 \%$ \\
\hline No & 42 & $35.6 \%$ \\
\hline Total & 118 & $100 \%$ \\
\hline
\end{tabular}

Fuente: Encuesta-entrevista realizada a los profesionales de salud: ginecólogos y obstetras u obstetrices de la localidad de Tacna.

\section{DISCUSIÓN}

El desarrollo de una politica de salud intercultural es un tema reciente. Es asi que el diseño de estrategias que permitan el diálogo y comunicación entre los modelos médicos se encuentran receptivos a la creatividad de quienes van a ejecutar las acciones en salud intercultural. La experiencia internacional ha demostrado la existencia de principios conceptuales que sustentan el desarrollo de modelos pluriculturales en salud. Entre estos se encuentra el de reconocer a los sistemas médicos como complejos modelos de pensamiento y conducta, los cuales constituyen respuestas culturales al fenómeno universal de la enfermedad.

Podemos señalar que es momento de que las universidades de nuestro pais, brinden obligatoriamente una formación con enfoque intercultural a los profesionales y se mejoraria el proceso de comunicación entre los usuarios y los proveedores de salud, hecho que no sólo debe contemplar al lenguaje como acto comunicativo sino también al conocimiento sociocultural de los pacientes. En un contexto de salud intercultural, ambos ejes conceptuales son fundamentales y se basan en los actuales principios de bioética médica expresado en el respeto a la autonomia de los pacientes y a su derecho a decidir libre e informadamente en el marco de la cultura propia y dejar de imponer la nuestra.

\section{CONCLUSIONES}

1. Los profesionales de la salud ginecólogos y obstetrices u obstetras, manejan adecuadadamente conceptos de interculturalidad, la mayor parte de ellos no han recibido formación universitaria con elementos interculturales a pesar de ello, consideran que la atención con enfoque intercultural es un derecho, una necesidad y que se justifica para nuestra población. Asi mismo reconocen que la condición étnica genera inequidad y discriminación en la atención de salud, muchas veces por la falta de conocimiento de las culturas quechuas y aymaras.

2. Los elementos que mayormente dificultan el acceso de la población indigena a los servicios de salud se deben a la falta de preparación del profesional para brindar atención con enfoque intercultural, al maltrato al usuario y a los mitos y creencias propios de nuestra población.

3. Existe un adecuado conocimiento de las normas técnicas para la atención del parto vertical, a pesar de ello no se ha implementado, teniendo como principales causas la falta de capacitación, motivación e inadecuada infraestructura. 


\section{BIBLIOGRAFÍA}

Cueto, Marcos: Salud, Cultura y Sociedad en América Latina. IEP/OPS, Lima, 1996. Estudios Históricos.

Degregori, Carlos Iván: No hay País más Diverso. Compendio de Antropología Peruana. EP. Primera Edición. Lima, Mayo 2000.

Huber, Ludwing: Consumo, Cultura e Identidad en el Mundo Globalizado: Estudios de Casos en los Andes. IEP Lima 2002.

Ibacache Burgos,Jaime. Servicio de Salud Araucanía Sur.URL: http://www.xs4all.nl/ rehue/artiba1.html

MINSA, Oficina de Estadistica e Informática, programa de fortalecimiento de servicio y salud, segundo censo de infraestructura y recursos humanos. Lima: MINSA - OEIPESS, 1997.

Planas Toledo, Maria Elena, Antropología Nutricional en el Pueblo Joven de Tahuantinsuyo. Lima - Perú. Del Informe presentado en 1997.

Pedersen, Duncan; Pobreza y Racismo en Poblaciones Amerindias. 1999

"Salud pública." Enciclopedia® Microsof $₫$ Encarta 2001. ( 1993-2000 Microsoft Corporation. Reservados todos los derechos.

Theidon, Kimberly y Ponicano del Pino, Informe Final: Un Estudio de Recuperacion Comunal. Ayacucho, Junio, 1997. 